EPJ Web of Conferences 37, 01007 (2012)

DOI: $10.1051 /$ epjconf/20123701007

(C) Owned by the authors, published by EDP Sciences, 2012

\title{
The hadronic light-by-light contribution to the muon anomalous magnetic moment and renormalization group for EFT
}

\author{
Johan Bijnens $^{\mathrm{a}}$ and Mehran Zahiri Abyaneh
}

Dept. of Astronomy and Theoretical Physics, Lund University, Sölvegatan 14A, 22362 Lund, Sweden

\begin{abstract}
We give a short overview of the theory of the muon anomalous magnetic moment with emphasis on the hadronic light-by-light and the pion loop contribution. We explain the difference between the hidden local symmetry and full VMD pion loop and discuss leading logarithms in the anomalous sector of 2-flavour chiral perturbation theory.
\end{abstract}

\section{The muon anomalous magnetic moment}

In this section we give a short overview of the present status of the theory behind the muon anomalous magnetic moment and a few new results on the pion loop contribution to the light-by-light part.

Experiment and theory use the anomaly $a_{\mu} \equiv\left(g_{\mu}-2\right) / 2$. BNL E821 [1] dominates the world average [2] given in Tab. 1. The standard model prediction is a bit off. The prediction and its main parts are listed in Table 1. For definiteness we quote numbers and errors of [2], but there is agreement on all numbers except on the hadronic light-by-light (HLBL) part. The difference is given in the last line of Tab. 1 with errors added quadratically. The experiment will move to Fermilab with an expected inprovement of a factor of four. Theory thus needs to improve. The discrepancy has created a lot of excitement since many BSM models can predict a value in this range but often a lot more or a lot less. The value of $a_{\mu}$ provides a major constraint on many BSM models. Reviews of all aspects are [3,4].

\subsection{QED, Electroweak and Hadronic Vacuum Polarization}

The QED contribution is well known. The first term is due to Schwinger. The first three terms are known analytically. the fourth is a full numerical calculation and the fifth is an estimate. With $\bar{\alpha}=\alpha / \pi$,

$$
a_{\mu}^{\mathrm{QED}}=0.5 \bar{\alpha}+0.765857410(27) \bar{\alpha}^{2}+24.05050964(43) \bar{\alpha}^{2}+130.8055(80) \bar{\alpha}^{2}+663(20) \bar{\alpha}^{2}+\cdots
$$

Kinoshita and collaborators played a major role in evaluating all contributions numerically. The QED value in Tab. 1 uses $\alpha$ from the electron magnetic moment. The third order contribution is dominated by the unexpectedly large leptonic light-by-light (LLBL) contribution [5]. The Schwinger diagram is shown in Fig. 1a and the LLBL diagram in Fig. 1b with its part of the 24.05 QED third order in (1).

A typical one-loop electroweak diagram is Fig. 2a. Two-loop corrections are large due to large, partly hadronic, logarithms in diagrams like Fig. 2b, (triangle) anomaly in (muon) anomaly [8,9].

$$
10^{10} a_{\mu}^{\mathrm{EW}}=19.48[1-\mathrm{loop}]-4.07(0.10)(0.18)[2-\text { loop }]=15.4(0.1)(0.2)(\text { triangle })(\text { Higgs mass }) .
$$

The remaining relevant contributions in the standard model are all hadronic. The largest is the hadronic vacuum polarization (HVP). The bare quark-loop has large gluonic corrections and needs to

\footnotetext{
a e-mail: bijnens@thep.lu.se
} 
Table 1. Overview of results.

\begin{tabular}{lrr}
\hline $10^{10} a_{\mu}$ & value & error \\
\hline exp & 11659208.9 & 6.3 \\
\hline theory & 11659180.2 & 4.9 \\
\hline QED & 11658471.8 & 0.0 \\
EW & 15.4 & 0.2 \\
LO Had & 692.3 & 4.2 \\
HO HVP & -9.8 & 0.1 \\
HLBL & 10.5 & 2.6 \\
\hline difference & 28.7 & 8.1 \\
\hline
\end{tabular}

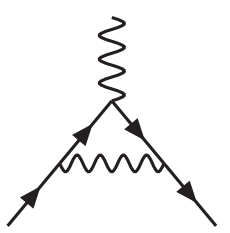

(a)

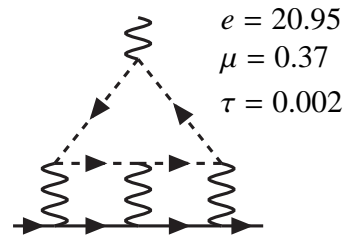

(b)

Fig. 1. Examples of QED contributions. (a) The contribution calculated by Schwinger. (b) The leptonic light-by-light contribution.

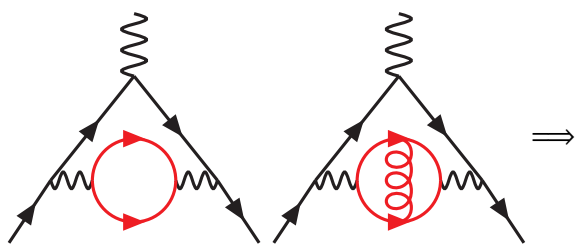

Fig. 3. The lowest-order hadronic vacuum-polarization (HVP) contribution to $a_{\mu}$. We need to sum all higher order QCD corrections.
Table 2. The different parts of the HLBL contribution.

\begin{tabular}{ccc}
\hline & BPP [6] & PdRV [7] \\
\hline pseudo-scalar & $(8.5 \pm 1.3) \cdot 10^{-10}$ & $(11.4 \pm 1.3) \cdot 10^{-10}$ \\
axial-vector & $(0.25 \pm 0.1) \cdot 10^{-10}$ & $(1.5 \pm 1.0) \cdot 10^{-10}$ \\
quark-loop & $(2.1 \pm 0.3) \cdot 10^{-10}$ & - \\
scalar & $(-0.68 \pm 0.2) \cdot 10^{-10}$ & $(-0.7 \pm 0.7) \cdot 10^{-10}$ \\
$\pi K$-loop & $(-1.9 \pm 1.3) \cdot 10^{-10}$ & $(-1.9 \pm 1.9) \cdot 10^{-10}$ \\
\hline errors & linearly & quadratically \\
sum & $(8.3 \pm 3.2) \cdot 10^{-10}$ & $(10.5 \pm 2.6) \cdot 10^{-10}$ \\
\hline
\end{tabular}

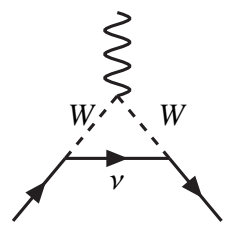

(a)

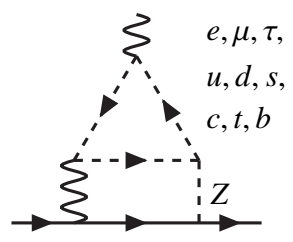

(b)
Fig. 2. The electroweak contributions. (a) A typical 1-loop diagram. (b) An example of a triangle anomaly diagram appearing at 2-loop order.

be done to all orders in $\alpha_{S}$ as depicted in Fig. 3. This contribution can be related to experiment via

$$
a_{\mu}^{\mathrm{LOhad}}=\frac{1}{3}\left(\frac{\alpha}{\pi}\right)^{2} \int_{m_{\pi}^{2}}^{\infty} d s \frac{K(s)}{s} R^{(0)}(s),\left.\quad R^{(0)}(s) \equiv \frac{\sigma\left(e^{+} e^{-} \rightarrow \text { hadrons }\right)}{\sigma\left(e^{+} e^{-} \rightarrow \mu^{+} \mu^{-}\right)}\right|_{\text {bare }} .
$$

The precise definition of bare led to some confusion between theory and experiment and there were experimental discrepancies. A representative value is given in Tab. 1, see [2-4] for references and discussion. At higher orders in $\alpha$ two types of hadronic contributions are relevant. Those with two insertions of the HVP, as in Fig. 4, see [2-4], can be evaluated from $R^{(0)}(s)$ and the HLBL contribution is discussed in Sect. 1.2. Values are again given in Tab. 1.

\subsection{Hadronic Light-by-Light}

The HLBL contribution is depicted in Fig. 5. The muon and photon lines are the well known part. The blob needs to be filled with hadrons and QCD. The trouble is that low- and high-energy are very mixed and a double counting of different hadron/quark contributions needs to be avoided. A workshop at INT, Seattle [10] provides a good overview of the situation. A start on separating the different parts is by studying at which orders in the large number of colours, $N_{c}$, and chiral, $p$, expansion, contributions start [11]. The pion loop is $1, p^{4}$, pion-exchange is $N_{c}, p^{6}$ and all others start at $N_{c}, p^{8}$. This separation was used to do a full calculation by two independent groups, [6] and [12]. The latter 


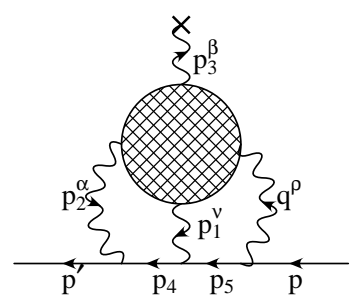

Fig. 5. The hadronic light-by-light (HLBL) contribution to $a_{\mu}$.

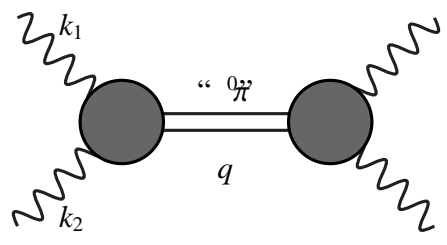

Fig. 6. The pseudo-scalar exchange diagram. The blobs denote the formfactor $F\left(q^{2}, k_{1}^{2}, k_{2}^{2}\right)$.

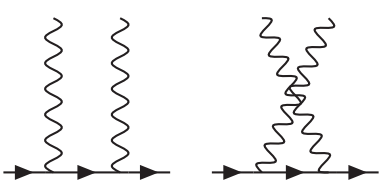

Fig. 7. The tree level diagrams that contract to the axial vector current in the limit $P_{1}^{2} \approx P_{2}^{2} \gg$ $Q^{2}$.

used purely hadronic exchanges and added a quark-loop with a VMD suppression as well as the pion loop in hidden local symmetry (HLS) model. They studied the dependence on the vector meson mass to determine the important energy regions. The former used the extended Nambu-Jona-Lasinio model of $[13,14]$ as a basis while repairing its worst shortcomings. The advantage is that this model has quarks and automatically generates pseudo-scalars, vectors and axial-vectors with a reasonable description. The scalars are unphysical but describe to some extent $\pi \pi$ scattering effects. In addition to the large $N_{c}$ ENJL contributions they added the pion-loop with phenomenological VMD in all photon legs and the short distance quark-loop. They studied the cut-off dependence of the various contributions. A sign mistake in the pion-exchange by both groups was discovered by [15]. Note that since $[6,12]$ no new full calculation was done.

The HLBL contribution in all detail is given by, momenta and indices as in Fig. 5,

$$
a_{\mu}^{\mathrm{HLBL}}=\frac{-e^{6}}{48 m_{\mu}} \operatorname{tr} \int \frac{\mathrm{d}^{4} p_{1} \mathrm{~d}^{4} p_{2}}{(2 \pi)^{8}} \frac{\gamma_{\alpha}\left(\not p_{4}+m_{\mu}\right) \gamma_{\nu}\left(\not p_{5}+m_{\mu}\right) \gamma_{\rho}}{q^{2} p_{1}^{2} p_{2}^{2}\left(p_{4}^{2}-m_{\mu}^{2}\right)\left(p_{5}^{2}-m_{\mu}^{2}\right)}\left[\frac{\delta \Pi^{\rho v \alpha \beta}\left(p_{1}, p_{2}, p_{3}\right)}{\delta p_{3 \lambda}}\right]\left(\not p+m_{\mu}\right)\left[\gamma_{\lambda}, \gamma_{\beta}\right]\left(\not p+m_{\mu}\right) \text {. }
$$

The main object is the four point function of four electromagnetic currents $V_{i}^{\mu}(x) \equiv \sum_{i} Q_{i}\left[\bar{q}_{i}(x) \gamma^{\mu} q_{i}(x)\right]$,

$$
\Pi^{\rho v \alpha \beta}\left(p_{1}, p_{2}, p_{3}\right) \equiv i^{3} \int \mathrm{d}^{4} x \int \mathrm{d}^{4} y \int \mathrm{d}^{4} z e^{i\left(p_{1} \cdot x+p_{2} \cdot y+p_{3} \cdot z\right)}\left\langle 0\left|T\left(V_{a}^{\rho}(0) V_{b}^{v}(x) V_{c}^{\alpha}(y) V_{d}^{\beta}(z)\right)\right| 0\right\rangle
$$

and we used $\Pi^{\rho v \alpha \lambda}\left(p_{1}, p_{2}, p_{3}\right)=-p_{3 \beta}\left(\delta \Pi^{\rho v \alpha \beta}\left(p_{1}, p_{2}, p_{3}\right) / \delta p_{3 \lambda}\right)$ which allows to calculate directly at $p_{3}=0$ and makes the integrals more convergent [5].

The general $\Pi^{\rho v \alpha \lambda}$ contains 138 different Lorentz structures of which 32 contribute to $a_{\mu}$ [6]. Using the gauge invariance relations $q_{\rho} \Pi^{\rho v \alpha \beta}=p_{1 v} \Pi^{\rho v \alpha \beta}=p_{2 \alpha} \Pi^{\rho v \alpha \beta}=p_{3 \beta} \Pi^{\rho v \alpha \beta}=0$ the 132 can be reduced to 43 structures $[16,17]$ that after $p_{3} \rightarrow 0$ depend on $p_{1}^{2}, p_{2}^{2}, q^{2}$. There are 8 integrals in (4) and most evaluations have rotated the integrations to Euclidean space. Artefacts in models are smeared out there and the separation of long and short distances becomes easier. Three of the integrals are trivial and a new development is that of the remaining five two can be done using the Gegenbauer polynomial techniques $[4,15,17]$. So in the end integrals over $P_{1}^{2}=-p_{1}^{2}, P_{2}^{2}=-p_{2}^{2}, Q^{2}=-q^{2}$ remain.

To visually see the contribution of various quantities different scales we introduce [16]

$$
a_{\mu}^{\mathrm{X}}=\int d l_{P_{1}} d l_{P_{2}} a_{\mu}^{\mathrm{XLL}}=\int d l_{P_{1}} d l_{P_{2}} d l_{Q} a_{\mu}^{\mathrm{XLLQ}}, \quad \text { with } \quad l_{P}=\ln (P / \mathrm{G} e V) .
$$

The contributions of type $X$ at a given scale $P_{1}, P_{2}, Q$ are directly proportional to the volume under the surface when $a_{\mu}^{\mathrm{XLL}}$ and $a_{\mu}^{\mathrm{XLLQ}}$ are plotted versus the energies on a logarithmic scale.

The main contribution is pseudo-scalar exchange, $\pi^{0}$ (and $\eta, \eta^{\prime}$ ), depicted in Fig. 6. Here one has to model the form-factor $F\left(q^{2}, k_{1}^{2}, k_{2}^{2}\right)$ including the dependence on how off-shell the pion is $[4,6$, 12]. Treating it is pointlike gives a logarithmic divergence [12] which can be evaluated using chiral perturbation theory (ChPT) $[18,19]$. In [6] $\pi^{0}$-exchange was found to be essentially saturated at a scale of $1 \mathrm{GeV}$ and $10^{10} a_{\mu}^{\pi^{0}}=5.9$. Including $\eta, \eta^{\prime}$ exchange leads to the value listed under pseudo-scalar and BPP in Tab. 2. All models except [20] give basically a compatible value for $10^{10} a_{\mu}^{\pi^{0}}$ with 6.27 for the 


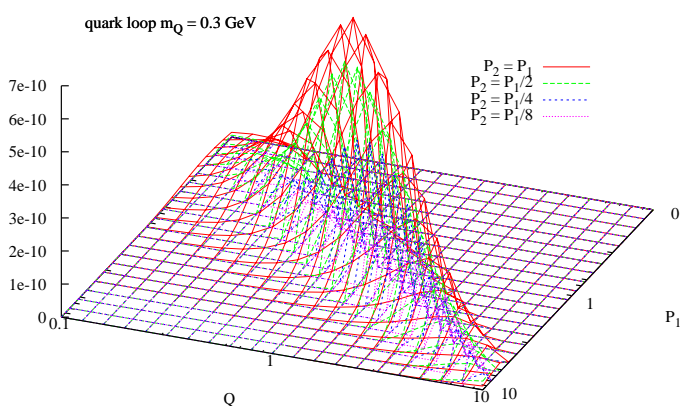

Fig. 8. The momentum distribution of the pure quarkloop contribution for various ratios of $P_{2} / P_{1}$.

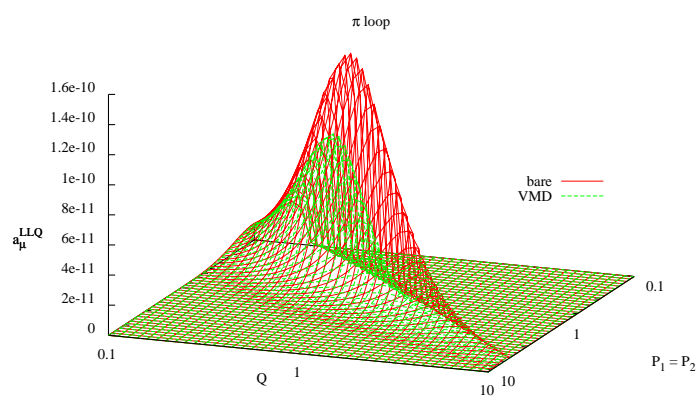

- Fig. 9. The momentum distribution of the bare and the pure VMD pion-loop contribution for $P_{2}=P_{1}$.

nonlocal quark model [21], 5.75 for a Dyson-Schwinger equations based approach [22], (5.8 - 6.3) for a hadronic modeling with two vectors and some short-distance constraints [15], 6.54 for a form-factor inspired by AdS/QCD [23], (6.5-7.1) for the chiral quark model [24], 7.2 \pm 1.2 with an extra constraint on the form-factor [25] and 7.5 from a direct AdS/QCD calculation including pion excitations [26].

A new development was the short-distance constraint on the region $P_{1}^{2} \approx P_{2}^{2} \gg Q^{2}$ by [20]. Here one uses the operator product expansion of two vector currents to relate $\Pi^{\rho v \alpha \beta}$ to a matrix-element of an axial current. [20] implemented this constraint by setting one of the form-factors in Fig. 6 to 1, i.e. pointlike. They obtained a value of $10^{10} a_{\mu}^{\pi^{0}}=7.7$ which with including $\eta, \eta^{\prime}$ leads to the number in Tab. 2 quoted under pseudo-scalar and PdRV. The OPE expansion comes from the diagrams of Fig. 7 so one expects that approaches involving the (short-distance) quark-loop do include this [7,21]. The distribution over energy scales for the two cases is shown in Figs. 5 and 7 of [16].

Axial-vector exchange is treated in the same way. [20] found an enhancement over [6] from both their short-distance constraint and the mixing of the two axial-vector nonets giving the difference between the two numbers for that contribution in Tab. 2. The ENJL model of [6] gave an estimate for scalar exchange as well.

The pure quark-loop contribution is known analytically [27] but estimates are already much older than that. Using $300 \mathrm{MeV}$ for the quark-mass one obtains $10^{10} a_{\mu}^{Q L} \approx 5$, with $240 \mathrm{MeV}$ [24] obtains about 8. The contribution over energies is about half below one $\mathrm{GeV} \mathrm{[6]} \mathrm{as} \mathrm{can} \mathrm{be} \mathrm{seen} \mathrm{in} \mathrm{Fig.} \mathrm{8.} \mathrm{[6]}$ used ENJL at low energies and a pure quark-loop with a heavy quark-mass as cut-off at high energies and found good stability versus the cut-off with the result quoted in Tab. 2. A much larger value was found in the DSE approach [22] but this calculation needs confirmation.

The last contribution is the one leading in ChPT, the charged pion, and a small kaon, loop. The bare pion loop gives a large contribution of $10^{10} a^{\pi-\text { loop }}=-4.1$ but this is expected to be too large. Several chirally invariant models were used, the HLS approach [12] which gave -0.45 and the VMD inspired approach [6] which gave -1.9 , an exact VMD approach gives $-1.6[6,12,17]$. One can derive from the OPE of two vector currents also a short distance relation for the $\gamma^{*} \gamma^{*} \pi \pi$ process which the HLS and the bare vertex do not satisfy while the VMD inspired approaches do [17]. The distribution over momenta of the contributions is shown in Fig. 9 for the bare and the pure VMD case and in Fig. 10 for the HLS and pure VMD. Notice how the large momentum contributions are cut-off in both the VMD and HLS case and the source for the large difference between VMD and HLS is the negative contribution at larger momenta for the HLS indicating that the VMD result is probably more correct.

[28] calculated the pion-loop four-point function $\Pi^{\rho v \alpha \beta}$ at very low momenta in ChPT. They found that for $P_{1}, P_{2}, P_{3}, Q \ll m_{\pi}$ there are indications that the effect due to $L_{9}$ and $L_{10}$ which in the HLS and VMD models is only partially taken into account could be important. We show the pure VMD result with the pion loop result including the effect of $L_{9}$ and $L_{10}$ for scales up to $500 \mathrm{MeV}$ in Fig. 11 . We did not take the limit $P_{1}, P_{2}, P_{3}, Q \ll m_{\pi}$. One sees indeed an enhancement of $10 \%$ due $L_{9}, L_{10}$. The full contribution to $a_{\mu}$ at that order in ChPT is divergent.

We conclude like $[4,6,12,7]$ that $a^{\mathrm{HLBL}} \approx(10 \pm 3) \cdot 10^{-10}$ where the exact central value and error are somewhat subjective. 


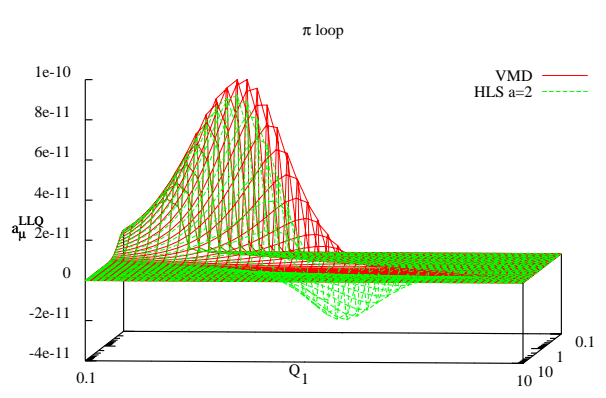

Fig. 10. The momentum distribution of the pure VMD and HLS pion-loop contribution for $P_{2}=P_{1}$.

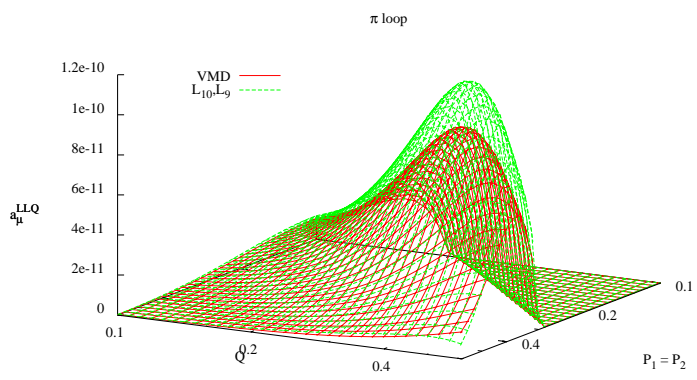

Fig. 11. The momentum distribution of the pure VMD pion-loop and the bare pion loop with $L_{9}, L_{10}$ effect included, contribution for $P_{2}=P_{1}$.

\section{Renormalization group for EFT}

Take an observable $F$ that depends on a single scale $M$. The dependence on this scale in quantum field theory (QFT) is typically logarithmic

$$
F(M)=F_{0}^{0}+F_{1}^{1} L+F_{0}^{1}+\sum_{n=2, \infty} \sum_{m=0, n} F_{m}^{n} L^{m}, \quad L=\ln (\mu / M) .
$$

The leading logarithms, the terms $F_{m}^{m} L^{m}$, are easier to calculate than the full result. This follows from two facts. The dependence of any observable on the subtraction scale $\mu$ vanishes, $\mu d F / d \mu=0$ and ultraviolet divergences in QFT are local. In renormalizable QFT leading logarithms can be described by a running coupling. This can be proven directly from the renormalization group, but relies on the fact that in a renormalizable theory counter-terms are of the same form at every order. It implies that the leading logarithms are calculable by a one-loop calculation. The counter-terms for an effective lowenergy theory, e.g. ChPT, differ at every order. However, Weinberg [29] pointed out that the two-loop leading logarithms can still be calculated from a one-loop calculation. The all order generalization was proven using beta-functions [30] and diagrams [31] . The main underlying observation is that at $n$-loop order all divergences must cancel. Using dimensional regularization with $d=4-w$ the coefficients of

$$
\left\{1 / w^{n}, \log \mu / w^{n-1}, \log ^{2} \mu / w^{n-2}, \ldots, \log ^{n-1} / w\right\} ;\left\{1 / w^{n-1}, \log \mu / w^{n-2}, \ldots, \log ^{n-2} \mu / 2\right\} ; \ldots
$$

must cancel. The first set of conditions allows to prove that all leading logs can be determined from one-loop diagrams, the second set that the subleading logs can be had from two-loop diagrams, etc..

The observation [31] that the needed Lagrangians at each order do not need to be minimal, allows them to be computer generated. The number of diagrams increases fast with order, e.g. mass at six loops requires 303 . The size of each diagram grows even faster. The leading logarithms for the mass in the massive $O(N)$ model were calculated to five loops in [31], for the decay constant and vev to the same order and for the vector and scalar form-factors as well as meson-meson scattering to four-loops in [32]. [31,32] also discussed the large $N$ limit for these quantities. The mass, decay constant and vector form-factor were pushed to six loops in [33]. [33]s main purpose was including the anomaly for the massive $O(3)$ model, i.e. two-flavour ChPT. The amplitude for $\pi^{0} \rightarrow \gamma^{*}\left(k_{1}\right) \gamma^{*}\left(k_{2}\right)$ is

$$
A_{\pi \gamma^{*} \gamma^{*}}=\epsilon_{\mu \nu \alpha \beta} \varepsilon_{1}^{* \mu}\left(k_{1}\right) \varepsilon_{2}^{* v}\left(k_{2}\right) k_{1}^{\alpha} k_{2}^{\beta} F_{\pi \gamma \gamma}\left(k_{1}^{2}, k_{2}^{2}\right) \quad F_{\pi \gamma \gamma}\left(k_{1}^{2}, k_{2}^{2}\right)=\frac{e^{2}}{4 \pi^{2} F_{\pi}} \hat{F} F_{\gamma}\left(k_{1}^{2}\right) F_{\gamma}\left(k_{2}^{2}\right) F_{\gamma \gamma}\left(k_{1}^{2}, k_{2}^{2}\right) .
$$

$\hat{F}$ is there for on-shell photons; $F_{\gamma}\left(k^{2}\right)$ is the form-factor for one off-shell photon and $F_{\gamma \gamma}$ is the nonfactorizable part when both photons are off-shell. The leading logarithms to six loops [33] are numerically

$$
\hat{F}=1+0-0.000372+0.000088+0.000036+0.000009+0.0000002+\cdots
$$

showing extremely good convergence. The form-factor $F_{\gamma}\left(k^{2}\right)$ also converges well but here the leading logarithms are known to be only a small part. The nonfactorizable part $F_{\gamma \gamma}$ only starts at three-loop 
order (could have started at two) and in the chiral limit only starts at four-loops. The leading logarithms thus predict this part to be fairly small.

Similarly, the leading logarithms for the $\gamma 3 \pi$ vertex are small and give a good convergence with

$$
F_{0}^{3 \pi L L}=(9.8-0.3+0.04+0.02+0.006+0.001+\cdots) \mathrm{GeV}^{-3} .
$$

\section{Acknowledgements}

Supported in part by the European Community SP4-Capacities (HadronPhysics3, Grant Agreement n. 283286) and the Swedish Research Council grants 621-2011-5080 and 621-2010-3326.

\section{References}

1. G. W. Bennett et al. [Muon G-2 Collaboration], Phys. Rev. D 73, (2006) 072003

2. K. Nakamura et al. [Particle Data Group Collaboration], J. Phys. G 37, (2010) 075021

3. J. P. Miller, E. de Rafael and B. L. Roberts, Rept. Prog. Phys. 70, (2007) 795

4. F. Jegerlehner and A. Nyffeler, Phys. Rept. 477, (2009) 1

5. J. Aldins, T. Kinoshita, S. J. Brodsky and A. J. Dufner, Phys. Rev. D 1, (1970) 2378

6. J. Bijnens, E. Pallante and J. Prades, Phys. Rev. Lett. 75, (1995) 1447 [Erratum-ibid. 75, (1995) 3781]; Nucl. Phys. B 474, (1996) 379; Nucl. Phys. B 626, (2002) 410

7. J. Prades, E. de Rafael and A. Vainshtein,

8. A. Czarnecki, W. J. Marciano and A. Vainshtein, Phys. Rev. D 67, (2003) 073006

9. M. Knecht, S. Peris, M. Perrottet and E. De Rafael, JHEP 0211, (2002) 003

10. http://www . int. washington. edu/PROGRAMS/11-47w/

11. E. de Rafael, Phys. Lett. B 322, (1994) 239

12. M. Hayakawa, T. Kinoshita and A. I. Sanda, Phys. Rev. Lett. 75, (1995) 790; Phys. Rev. D 54, (1996) 3137; M. Hayakawa and T. Kinoshita, Phys. Rev. D 57, (1998) 465 [Erratum-ibid. D 66, (2002) 019902]

13. J. Bijnens, C. Bruno and E. de Rafael, Nucl. Phys. B 390, (1993) 501

14. J. Bijnens, Phys. Rept. 265, (1996) 369

15. M. Knecht and A. Nyffeler, Phys. Rev. D 65, (2002) 073034

16. J. Bijnens and J. Prades, Mod. Phys. Lett. A 22, (2007) 767

17. J. Bijnens and M. Zahiri Abyaneh, work in progress, M. Zahiri Abyaneh, master thesis, LU TP 12-26, arXiv:1208.2554 [hep-ph]

18. M. Knecht, et al., Phys. Rev. Lett. 88, (2002) 071802

19. M. J. Ramsey-Musolf and M. B. Wise, Phys. Rev. Lett. 89, (2002) 041601

20. K. Melnikov and A. Vainshtein, Phys. Rev. D 70, (2004) 113006

21. A. E. Dorokhov, W. Broniowski, Phys. Rev. D78, (2008) 073011

22. T. Goecke, C. S. Fischer and R. Williams, Phys. Rev. D 83, (2011) 094006

23. L. Cappiello, O. Cata and G. D’Ambrosio, Phys. Rev. D 83, (2011) 093006

24. D. Greynat and E. de Rafael, JHEP 1207, (2012) 020

25. A. Nyffeler, Phys. Rev. D 79, (2009) 073012 [arXiv:0901.1172 [hep-ph]], these proceedings

26. D. K. Hong and D. Kim, Phys. Lett. B 680, (2009) 480

27. S. Laporta and E. Remiddi, Phys. Lett. B 301, (1993) 440

28. K. T. Engel, H. H. Patel and M. J. Ramsey-Musolf, arXiv:1201.0809 [hep-ph]

29. S. Weinberg, Physica A 96, (1979) 327

30. M. Büchler and G. Colangelo, Eur. Phys. J. C 32, (2003) 427

31. J. Bijnens and L. Carloni, Nucl. Phys. B 827, (2010) 237

32. J. Bijnens and L. Carloni, Nucl. Phys. B 843, (2011) 55

33. J. Bijnens, K. Kampf and S. Lanz, Nucl. Phys. B 860, (2012) 245 